MATEC Web of Conferences 22,03007 (2015)

DOI: $10.1051 /$ matec conf/ 20152203007

(C) Owned by the authors, published by EDP Sciences, 2015

\title{
Review of Diagnosis Technique for Equipment Faults and its Develop- ment Trend
}

\author{
Lihua Sun*, Hang Bai, Gang Zhao \& Xianda Wang \\ Troop 63981 of Peoples' Liberation Army, Wuhan, Hubei, China
}

\begin{abstract}
Modern control system is becoming larger and more complicated with each passing day and the possibility of system breakdown increases with it, so people eagerly need to set up fault diagnosis system to conduct real-time monitoring and fault diagnosis for production system and take necessary measures to improve its overall reliability and maintainability. This paper states the principles and basic approaches of diagnosis technique for equipment faults, introduces development phrase of fault diagnosis technique and points out future development trend in this field.
\end{abstract}

Keywords: fault diagnosis; development trend; diagnosis technique

\section{INTRODUCTION}

With the rapid development of modern industry and science technology, integrated scale, performance and composition of equipment are becoming increasingly complicated and the demand for overall reliability of equipment system is increasingly strict, not only each part of equipment itself correlative, but also close relationship among different equipment, with fault of one part mightily causing breakdown of whole system. Therefore, fault diagnosis technique plays an important role in repairing and maintaining equipment and gets people's huge attention. Equipment fault diagnose is comprehensive and interdisciplinary practical technology, achieving automatic detection, constant value, location and fault prediction of equipment fault diagnose.

\section{OVERVIEW OF FAULT DIAGNOSIS TECH- NIQUE}

\subsection{Principle of fault diagnosis technique}

Fault diagnosis technique also called condition-based technique is a kind of fault state of dynamic monitoring equipment system. When electronic instrument appears abnormal situation, it can timely analyze fault causes and provide customers with relevant information through concrete issues of archives automatically diagnosing faults. It is also a technology to predict future state of equipment and it is an effective measure to prevent accidents and also important basis for equipment maintenance. The basic principle of diagnosis technique for equipment fault is shown as Figure 1, including four working procedures which are fault detection, feature extract, state recognition and forecasting decision.

*Corresponding author: sunlihua55@163.com

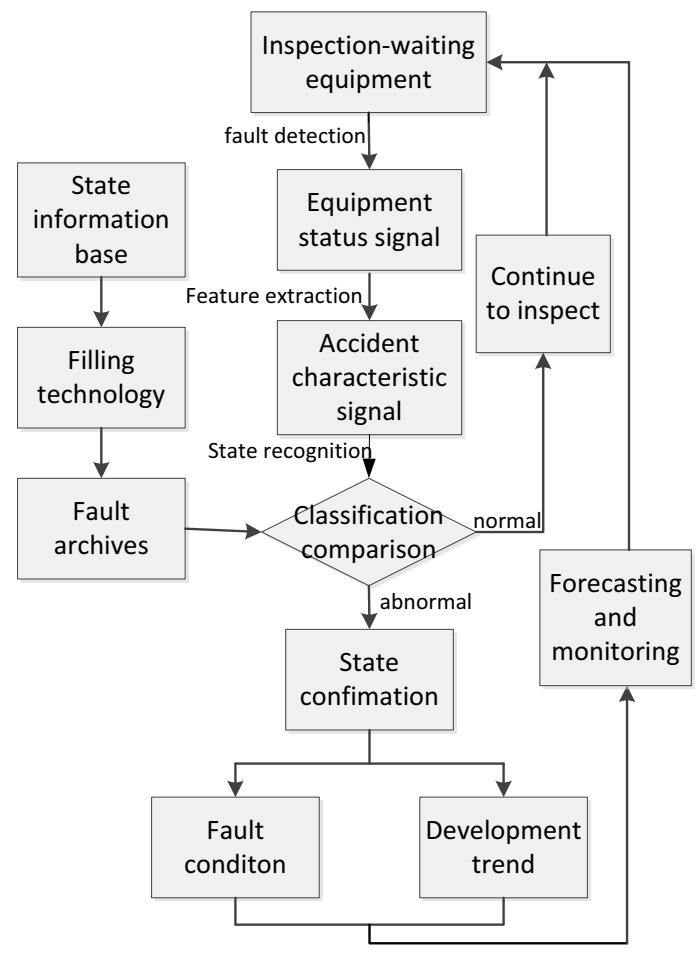

Figure 1. Working principle of diagnosis technique for equipment fault

\subsubsection{Fault detection}

It can generate signal that can reflect faults, and can constantly detect changes of variables though detecting system. In the nominal case, it thinks that these variables meet known pattern under an uncertainty and when any part of system goes wrong, these variables

This is an Open Access article distributed under the terms of the Creative Commons Attribution License 4.0, which permits unrestricted use, distribution, and reproduction in any medium, provided the original work is properly cited. 


\section{MATEC Web of Conferences}

diverge their nominal condition. It is used to judge faults usually according to system output or characteristics of estimated residuals of state variable.

\subsubsection{Feature extraction}

The feature extraction also called fault isolation can extract fault component in accordance with residual direction and structure and can extract fault signatures to form inspection-waiting pattern through magnifying, compressing, formal transformation and wiping out noise jamming and other means.

\subsubsection{State recognition}

It is used to conduct logical decision on time, size and site where faults happen. It can make a comparison and classify between inspection-waiting pattern and benchmark pattern, namely, equipment state is confirmed to be normal or not. It can compare equipment characteristic parameter or discriminant parameter achieved by signal processing to judge running status of machines. Therefore corresponding criteria and diagnostic strategies shall be formulated in a right way.

\subsubsection{Forecast decision}

It is used to judge equipment state. When state is normal, it can continue to monitor and with abnormal state, it shall find out faults to make countermeasures and can conduct trend analysis in accordance with trend where current signal predicts equipment state.

\subsection{Process of fault diagnosis technique}

Through fault phenomenon, users choose equipment that conduct fault diagnosis for inquiry test, analyze fault principle starting from fault symptoms and hypothetically deduce possible causes of generating fault phenomenon and finally verify fault points tenable or not by means of test. When hypothetical fault point that test verifies is not tenable, return to previous link to come up with new hypothetical deduction for verification. When new hypothetical deduction cannot be raised, we need to return to previous link for new analytical investigation. If it is still not ok, we continue to return to previous link for circulation until fault point is found. Flow chart of fault diagnosis is shown as Figure 2.

\section{DEVELOPMENT PHASE OF FAULT DIAGNO- SIS TECHNIQUE}

\subsection{Primitive diagnosis phase}

The end of $19^{\text {th }}$ century and beginning of $20^{\text {th }}$ century was infancy of fault diagnosis. Artificial method for fault detection and diagnosis is entirely used. Operators or expects at different areas make direct judgments by means of experience or using professional instruments to conduct field measurement for analytical judgments. This method is economical and practical in the fault diagnosis of simple equipment, however, its shortcomings is self-evident.

\subsection{Diagnosis phrase based on material lifetime analysis}

At the beginning of $20^{\text {th }}$ century to 1960 , owing to development and application of reliable theory, people can conduct fault analysis based on lifetime of hardware materials and take advantage of information provided by equipment to confirm fault components on the basis of the minority subordinate to the majority. The method usually uses triple or more components to finish the same function and also increases costs, structure and weight and others of system while improving system reliability and is unpractical for large-scale and complicated system.

\subsection{Diagnosis phrase based on analytical lengthiness}

This diagnosis began in the middle of 1960 uses functional lengthiness relationship of different parts of system to improve estimated technology to achieve lengthiness information to form residual because of sensor technology and takes advantage of different methods to dispose of residual to get fault information of system, making various diagnosis system and data measurements become relatively easy. It is current mainstream in this field with low cost and prone to engineering realization, along with the use of computers.

\subsection{Intelligent diagnosis phrase}

With the development of artificial intelligence tech-

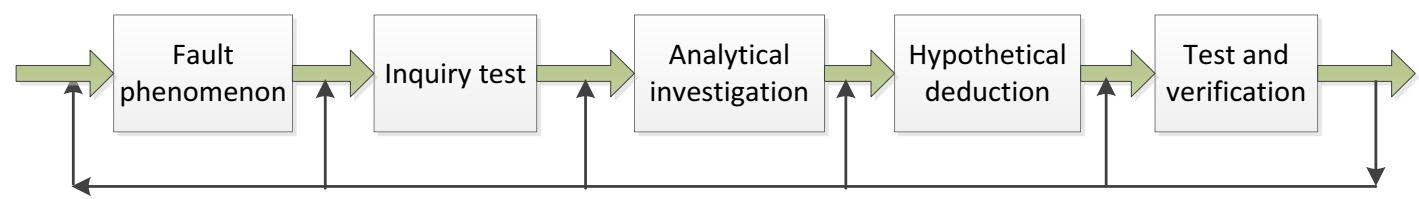

Figure 2. Flow chart of fault diagnosis 
ICETA 2015

nology, especially application of expert system in the field of fault diagnosis, this diagnosis is diagnostic procedure using new knowledge processing and knowledge inference instead of numerical technique and signal processing as its core. At present, intelligent diagnosis has become a new direction of diagnosis technique development.

\section{BASIC APPROACHES TO FAULT DIAGNOSIS TECHNIQUE}

There are three main approaches to fault diagnosis: fault diagnosis method based on analytical mode, based on signal processing and based on knowledge.

\subsection{Fault diagnosis method based on analytical mode}

This method needs to set up relatively precise mathematic model for diagnosed objects. Through comparing measurable information of diagnosed objects with prior information of system expressed by mathematic model, residual can be generated and this diagnosis conducts analyzing and dealing with residuals to achieve fault diagnosis technology.

\subsubsection{Method of state estimation}

Its basic idea is to use quantitative model and measurement signal of system to rebuild a certain measurable variable and to make the difference of estimated value of observer and measurable value of real system as residual and to conduct analytical processing to residual for detecting and isolating system faults. Therefore, key task is able to achieve precise mathematic module of system.

\subsubsection{Method of parameter estimation}

Its basic thought is to link faults with significant changes of system parameter and to use deviation between estimated value and normal value of parameter for detecting and isolating faults. Method of parameter estimation demands to find one-to-one relationship between model parameter and physical parameter and needs fully motivating in controlled process. Method of parameter estimation is much easier to isolate faults compared with method of state estimation.

\subsubsection{Method of equivalent space}

The basic idea is to use whether there is in agreement between actual measured value of system in-out and normal model for inspecting and isolating faults.

\subsection{Fault diagnosis method based on signal pro- cessing}

This method appears mainly aiming at analytical mathematic model of diagnostic objects which is difficult to set up and avoids difficulty of accurate model of diagnostic objects. It extracts characteristic value and directly analyzes measurable signals to detect faults, owing to strong adaption through various spectral analysis methods, correlation function and adaptive signal processing and other means.

\subsubsection{Detect fault diagnosis method based on Kull- back information principle}

Kullback information principle can measure system changes. Kullback information principle is compared with threshold values which can effectively detect faults while using unmodeled dynamic characteristics as soft boundary estimate. New evaluation indicator introduced to Kullback information principle to estimate unmodeled dynamic characteristics and decision scheme is rationally designed to achieve fault detection.

\subsubsection{Fault diagnosis method based on estimation of signal model.}

Its basic thought is to diagnose fault in line with changes of system physical parameter in a direct way. Using various methods and diagnosing each testing parameter as far as possible, this process is called local diagnosis. Combining results achieved by various diagnostic methods to get overall result of fault diagnosis is referred as global integrated diagnosis, achieving local-global integration scheme, which produces more precious and complete estimation and judgments than single information source. Global integrated diagnosis can solve low reliability of diagnosis exited in fault diagnosis by complex system to achieve fault detection.

\subsubsection{Fault diagnosis method based on wavelet transform}

Fault diagnosis method based on wavelet transform is a kind of time-scale analysis method, owning the feature of multi-resolution analysis. It can make system input and output signal conduct wavelet transform to obtain all singular points. It can use continuously wavelet transform to distinguish signal saltation and noise, retain corresponding singular points of system fault, can real-time detect faults online and has high sensitivity to achieve fault diagnosis of system.

\subsection{Knowledge-based fault diagnosis method}

This method introduces lots of information of diagnostic objects and can fully use expert diagnosis fault, especially large development space in nonlinear field. It does not need quantitative mathematic model of system and also overcomes disadvantages based on signal processing method. Knowledge-based method is mainly divided into quantitative model-based method and symptom-based method. Symptom-based method consists of neutral network method, fuzzy reasoning method, expert system method, pattern recognition method and fault tree method and so on. 


\section{MATEC Web of Conferences}

\subsubsection{Neutral network method}

Neutral network method owns ability of fitting continuous nonlinear function and self-learning from sample plate and its parallel processing and global role and makes it have strong advantage in dealing with nonlinear problem and online estimate, winning widespread high attention.

\subsubsection{Fuzzy reasoning method}

Fuzzy reasoning method confirms to people's natural consciousness, possesses superiority in dealing with such aspects as long time-delay, time varying as well as nonlinearity of complex system, overcomes the difficulties caused by uncertainty under its process, inaccuracy as well as noise and is suitable for system with smaller measured value and without achieving precise system. At present, the main diagnostic ideas consist of fault diagnosis method based on fuzzy model; residual evaluation system based on adaptive fuzzy threshold; residual evaluation system based on fuzzy clustering; residual evaluation system based on fuzzy reasoning and fault diagnosis method based on fuzzy pattern recognition.

\subsubsection{Expert system method}

Expert system method makes use of rich practical experience accumulated by experts, applies specialized knowledge in specific field and reasoning technology in artificial intelligence for solution, simulates experts to parse and to solve problems and explains its reasoning process and how to make an conclusion so as to give play to greater role and benefits from experts, widely used in theory and engineering. Expert system method usually consists of two main parts: knowledge base and inference engine. Knowledge base includes knowledge used by inference engine and utilizes the knowledge to make inference conclusion, thus making a response to users' inquiry. The expert system structure diagram is shown as Figure 3.

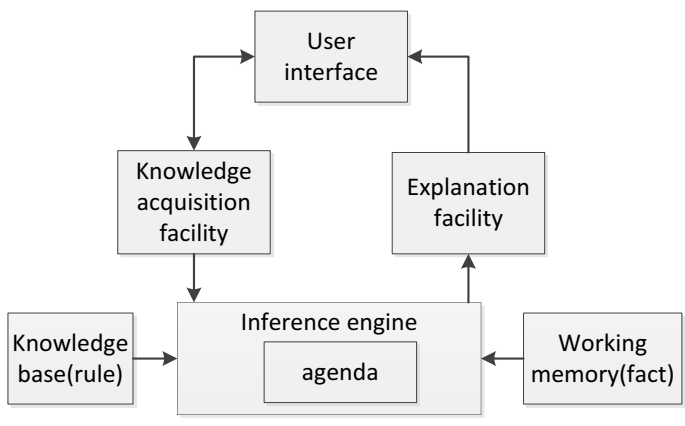

Figure 3. Expert system structure diagram

\subsubsection{Pattern recognition method}

Pattern recognition method for diagnosis can be divided into two phrases: off-line analysis and on-line diagnostics. Eigenvector constitution is frequency domain and time-domain signal parameter related to fault detection. Eigenvector samples train pattern clas- sifier in different faults and make decision for fault type by using linear discriminant function or K-nearest neighbor function while making diagnosis. This method has achieved great improvements in such aspects as graphic processing, computer vision and speech recognition and so on.

\subsubsection{Fault tree method}

Fault tree makes rational analysis for basic causes of breakdown in line with mutual relationship between system and equipment specific fault, then builds directed logic diagram describing breakdown from result to cause and makes total and partial detailed partition in arborescence for causes of system fault through logic diagram. This is a kind of graph deduction, owning such features as broad range and strong logic It also quantitatively calculates fault degree, probability and reason and so on. Basic structure of fault tree is shown as Figure 4.

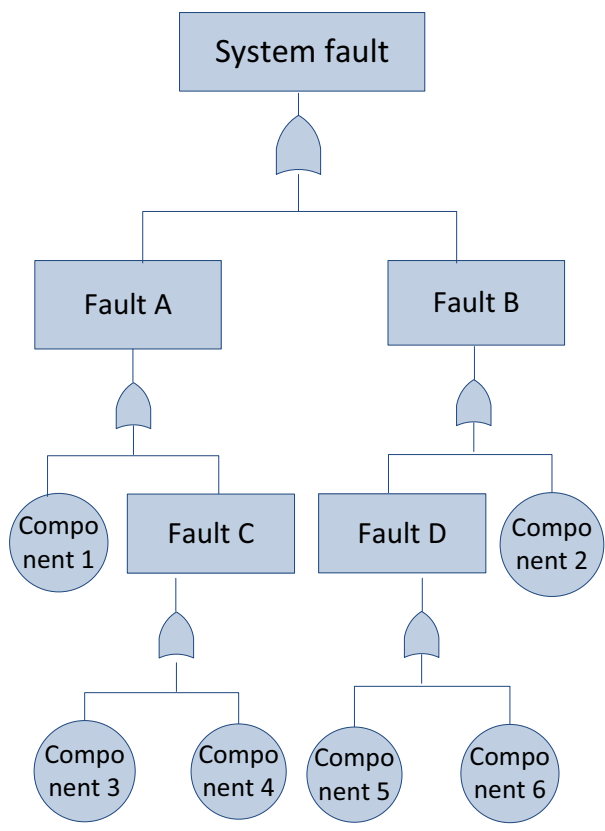

Figure 4. Basic structure of fault tree

Method based on qualitative model mainly pays much attention to precision of system description and can be divided into intelligent expert diagnosis system based on shallow knowledge, intelligent expert diagnosis system based on deep knowledge as well as intelligent expert hybrid diagnosis system. Shallow knowledge refers to experimental knowledge of filed experts. Deep knowledge is about structure, performance and function of diagnostic objects. Qualitative simulation is an important part of method based on qualitative model, uses qualitative variables showing system physical parameter and qualitative differential equation showing mutual relationship among various parameters to form constraint model, describes and 
simulates system structure and further confirms system state achieved from given original state, thus conducting fault diagnosis by qualitative reasoning.

\section{DEVELOPMENT TREND OF FAULT DIAG- NOSIS}

Fault diagnosis technique, with system and equipment for study and high and new technology for support, improves diagnosis precision and speed, confirms exact time and part of fault, estimates size and trend of fault and digests multiple engineering and technical system equipment and fields to form an interdisciplinary and comprehensive applied technology. Integration of equipment fault diagnosis technique with modern frontier science is development direction of equipment diagnosis technique, specific performance shown as follows:

\subsection{Integration of nonlinear principle and method with multiple sensor technology}

Information fusion of multi-sensor is a data processing process by using computer to make automatic analysis and synthesis for messages from multi-sensor in line with certain norm, conducts comprehensive and multi-angel supervision and maintenance for equipment so as to have integral and comprehensive understanding of equipment running status to achieve required decision and judgment. When equipment breaks down, it frequently shows nonlinear features, but with the improvement of chaos and fractal geometry method, this kind of problem will certainly be further solved.

\subsection{Intelligence}

Modern intellectual technology includes expert system, neutral network, evolutionary computation as well as fuzzy logic and so on. Intelligent fault diagnosis takes full advantage of integrated computer which exited in equipment controlling each subsystem and collects necessary information. It can directly judge and fix position for simple fault and send test equipment for complex fault by means of data bus so that expert system within test equipment makes analysis and fixes position and deals with these messages in line with certain method.

\subsection{Combination of multiple diagnosis methods}

Hybrid diagnosis system combining different intellectual technologies is development trend of research for intelligent fault diagnosis. Its combination modes mainly consist of combination of expert system based on rules and neutral network, instance reasoning and neutral network, and combination of fuzzy logic, neutral network and expert system and so on. Combination of various modes achieves complementary advantages and is able to effectively judge fault source and fault information, greatly improving precision of fault diagnosis.

\subsection{Remote and networking}

With the development of network technique, building remote fault diagnosis and monitoring system based on network can achieve common diagnosis of multi experts and multi systems. Remote equipment fault diagnosis system of networking stores fault diagnosis knowledge and experience of equipment can respond to usage requirements of users from different monitoring sites. Different monitoring sites can build connection with same diagnostic center.

\section{CONCLUSION}

Diagnosis technique for equipment faults is a strong practical technology, widely used in various fields such as aviation, ship, chemical engineering, mechanical equipment and so on. Condition monitoring and fault diagnosis for large-scale complicated equipment can implement condition-based maintenance, ensure equipment precision, save maintenance costs, prevent accidents and effectively guarantee equipment safety, and simultaneously, can avoid environmental pollution caused by equipment accidents and bring larger economic benefit and better social benefit, owning practical value in engineering.

\section{REFERENCES}

[1] Wang Xin. 2014. The Fault Diagnosis Technology of Mechanical Equipment and Application. Beijing: China Coal Industry Publishing House.

[2] Sitao Wu \& Tommy W.S. Chow. 2004. Induction machine fault detection using SOM-based RBF neural networks. IEEE Transactions on Industrial Electronics.

[3] Rolf Isermann. 2005. Model-based fault-detection and diagnosis-status and applications. Annual Reviews in Control.

[4] Timothy L. Skvarenina. 2002. The Power Electronics Handbook. CRC Press LLC.

[5] Wang Quanxian. 2013. The Fault Diagnosis Technology of Mechanical Equipment. Wuhan: Huazhong University of Science and Technology Press.

[6] Wang Yonghua, Chen Huagang. 2014. Electrical Equipment Fault Diagnosis Technology. Beijing: Chinese Power Press.

[7] Morpurgo R, Mussi S. 2001. I-DSS: An intelligent diagnostic support system. Expert Systems.

[8] Jun Liao, Meng Joo Er, Lin Jianya. 2000. Application of a system for the automatic generation of fuzzy neural networks. Engineering Application of Artificial Intelligence.

[9] Yang Jun, Feng Zhengsheng, Zhang Xien, et al. 2001. Study on missile intelligent fault diagnosis system based on fuzzy NN expert system. Journal of Systems Engineering and Electronics. 


\section{MATEC Web of Conferences}

[10]Chrissanthi Angeli. 2001. Derek Atherton. A model-based method for an on-line diagnostic knowledge-based system. Expert Systems.

[11] Smola A, Scholkopf B. 1998. A tutorial on support vectoregression. Neuro COLT: Technical Report.

[12]Larsson, J.E. 2000. Avoiding human error. Proceedings of the International Conference on Control and Instrumentation in Nuclear Installations, Bristol, England.

[13] Achmad Widodo, Bo Suk Yang, Tian Han. 2006. Combination of independent component analysis and support vector machines for intelligent faults diagnosis of induction motors. Expert Systems with Applications.

[14] Escobet T, Feroldi D, Delira S. 2008. Model based fault diagnosis in PEM fuel cell systems. Journal of Power Sources.

[15]Yang Fuqiang. 2012. Application of non-destructive testing technology in coal mine safety inspection in mechanical equipment. Mining Machinery.

[16]Feng Tengfei, Yang Jingsheng. 2012. Application of fault analysis in equipment management. Science and Technology Enterprises.

[17] Yang Chao, Li Yitao. 2011. Status and development of intelligent diagnosis technology of mechanical equipment fault. Journal of East China Jiaotong University.

[18] Michael G P. 2008. Prognostics and Health Management of Electronics. New Jersey: John Wiley \& Sons. Inc. Hoboken.

[19]Fan J Q, Yao Q W. 2003. Nonlinear Time Series: Non-parametric and Parametric Methods. USA: Springer.

[20]Zhao Jiong, Zhou Qicai, Xiong Xiaolei. 2014. Technology of Remote Fault Diagnosis and Maintenance. Machinery Industry Press.

[21]Xia Hong. 2010. The Fault Diagnosis Technology of Equipment. Harbin Institute of Technology Press. 